PROCEEDINGS OF THE

AMERICAN MATHEMATICAL SOCIETY

Volume 124, Number 12, December 1996, Pages 3875-3884

S 0002-9939(96)03691-X

\title{
FIBONACCI NUMBERS, LUCAS NUMBERS AND INTEGRALS OF CERTAIN GAUSSIAN PROCESSES
}

\author{
LUDWIG BARINGHAUS
}

(Communicated by Wei-Yin Loh)

\begin{abstract}
We study the distributions of integrals of Gaussian processes arising as limiting distributions of test statistics proposed for treating a goodness of fit or symmetry problem. We show that the cumulants of the distributions can be expressed in terms of Fibonacci numbers and Lucas numbers.
\end{abstract}

\section{INTRODUCTION}

Let $X_{1}, \ldots, X_{n}, \ldots$ be a sequence of independent and identically distributed $d$-dimensional random (column) vectors with Fourier transform $\phi$. Let $\phi_{n}(t)=$ $\frac{1}{n} \sum_{k=1}^{n} \exp \left(i t^{\prime} X_{k}\right), t \in \mathbb{R}^{d}$, be the empirical Fourier transform of the first $n$ observation vectors. It follows from the Glivenko-Cantelli theorem that as $n \rightarrow \infty$, the $\phi_{n}$ converge almost surely to $\phi$ uniformly on compact subsets of $\mathbb{R}^{d}$. This gives the motivation for various statistical procedures based on empirical Fourier transforms. Let us concentrate on two applications. The first one is a goodness of fit problem. Assuming that the distribution of the $X_{k}$ is unknown, a suitable test statistic for testing the hypothesis that the $X_{k}$ have the $d$-variate unit normal distribution $N_{d}$ with density $f_{d}(x)=\left(\frac{1}{2 \pi}\right)^{d / 2} \exp \left(-|x|^{2} / 2\right), x \in \mathbb{R}^{d}$, and Fourier transform $\phi(t)=\exp \left(-|t|^{2} / 2\right), t \in \mathbb{R}^{d}$, where $|\cdot|$ denotes the Euclidean norm on $\mathbb{R}^{d}$, is

$$
T_{1 n}=n \int\left|\phi_{n}(t)-\exp \left(-|t|^{2} / 2\right)\right|^{2} d N_{d}(t) ;
$$

see Keller [10], considering the case $d=1$, or Baringhaus and Henze [3], treating the composite hypothesis of multivariate normality. As a second application we mention the problem of testing the hypothesis of symmetry, meaning that the random vectors $X_{k}$ and $-X_{k}$ have the same distribution. Then one may suggest the test statistic

$$
T_{2 n}=n \int\left[\operatorname{Im} \phi_{n}(t)\right]^{2} d Q(t),
$$

where $Q$ is taken to be a distribution symmetric about the origin; see Feuerverger and Mureika [9], considering the case $d=1$. Choosing $Q=N_{d}$ we have the

Received by the editors May 15, 1995.

1991 Mathematics Subject Classification. Primary 60E05; Secondary $11 B 35$.

Key words and phrases. Gaussian processes, Fibonacci numbers, Lucas numbers, integral equations, empirical Fourier transform, testing for normality, testing for symmetry. 
representations

$$
T_{\text {in }}=\int G_{\text {in }}(t)^{2} d N_{d}(t), \quad i=1,2,
$$

with

$$
G_{1 n}(t)=\frac{1}{\sqrt{n}} \sum_{k=1}^{n}\left(\cos \left(t^{\prime} X_{k}\right)+\sin \left(t^{\prime} X_{k}\right)-\exp \left(-|t|^{2} / 2\right)\right), \quad t \in \mathbb{R}^{d}
$$

and

$$
G_{2 n}(t)=\frac{1}{\sqrt{n}} \sum_{k=1}^{n} \sin \left(t^{\prime} X_{k}\right), \quad t \in \mathbb{R}^{d} .
$$

For unit normal $X_{k}$ the covariance functions $\rho_{i}(s, t)$ of the empirical processes $\left\{G_{i n}(t), t \in \mathbb{R}^{d}\right\}, i=1,2$, are

$$
\rho_{1}(s, t)=\exp \left(-|s-t|^{2} / 2\right)-\exp \left(-|s|^{2} / 2\right) \exp \left(-|t|^{2} / 2\right), \quad s, t \in \mathbb{R}^{d},
$$

and

$$
\rho_{2}(s, t)=\frac{1}{2}\left[\exp \left(-|s-t|^{2} / 2\right)-\exp \left(-|s+t|^{2} / 2\right)\right], \quad s, t \in \mathbb{R}^{d} .
$$

It will be demonstrated in the next section that in the case of $d$-variate unit normal $X_{k}$ the process $\left\{G_{i n}(t), t \in \mathbb{R}^{d}\right\}$ has limiting sample path continuous Gaussian processes $\left\{G_{i}(t), t \in \mathbb{R}^{d}\right\}$ with zero mean and covariance function $\rho_{i}$, implying weak convergence of the laws of $T_{i n}=\int G_{i n}(t)^{2} d N_{d}(t)$ to the laws of $T_{i}=\int G_{i}(t)^{2} d N_{d}(t)$. The present paper aims to study these limit laws. It will be seen in Section 3 and Section 4 that the cumulants can be expressed in terms of Fibonacci and Lucas numbers.

\section{Preliminaries}

In what follows $\left\{G_{n}(t)\right\}$ and $\rho$ stand for the sequence of processes $\left\{G_{1 n}(t)\right\}$ with covariance function $\rho_{1}$ or for the sequence of processes $\left\{G_{2 n}(t)\right\}$ with covariance function $\rho_{2}$. Let $K$ be any compact subset of $\mathbb{R}^{d}$. Denote by $\mathcal{C}(K)$ the separable Banach space of real valued continuous functions on $K$ with the supremum norm. $G_{n}$ restricted to $K$ can be regarded as a random element in $\mathcal{C}(K)$. Applying Theorem 3.1 of Csörgö [4] or a central limit theorem for Banach space valued random variables (see, e.g. Araujo and Giné [2], Corollary 7.17) we obtain the weak convergence of $\left\{G_{n}(t), t \in K\right\}$ to some zero mean Gaussian process $\{G(t), t \in K\}$ with covariance function $\left.\rho\right|_{K \times K}$. Adapting the results of Whitt [12] we get the weak convergence of the process $\left\{G_{n}(t), t \in \mathbb{R}^{d}\right\}$ to some zero mean Gaussian process $\left\{G(t), t \in \mathbb{R}^{d}\right\}$ with covariance function $\rho$. Here these processes are regarded as random elements in the separable Fréchet space of all real valued continuous functions on $\mathbb{R}^{d}$ endowed with the $\sigma$-algebra of Borel sets generated by the topology of uniform convergence on compacta. Applying the Continuous Mapping Theorem we see that given any compact subset $K$ in $\mathbb{R}^{d}$, the integrals $\int_{K} G_{n}(t)^{2} d N_{d}(t)$ converge in distribution to $\int_{K} G(t)^{2} d N_{d}(t)$. Given any $\eta>0, \epsilon>0$ we can choose some compact set $K \subset \mathbb{R}^{d}$ such that

$$
P\left(\int_{K^{c}} G_{n}(t)^{2} d N_{d}(t)>\epsilon\right) \leq \frac{1}{\epsilon} \int_{K^{c}} \rho(t, t) d N_{d}(t) \leq \eta
$$


for all $n$. From this it follows that the integrals $\int G_{n}^{2} d N_{d}(t)$ converge in distribution to $T=\int G(t)^{2} d N_{d}(t)$. To study the distribution of $T$ we introduce the Hilbert-Schmidt operator $B_{d}: L_{2}\left(\mathbb{R}^{d}, N_{d}\right) \rightarrow L_{2}\left(\mathbb{R}^{d}, N_{d}\right)$, defined by $\left(B_{d} f\right)(t)=$ $\int \rho(s, t) f(s) d N_{d}(s), t \in \mathbb{R}^{d}, f \in L_{2}\left(\mathbb{R}^{d}, N_{d}\right)$. Let $\left\{\lambda_{k}, e_{k}, k \geq 1\right\}$ be a system of eigenvalues and orthonormal eigenfunctions of $B_{d}$, that is, $\int e_{k}^{2} d N_{d}=1, \int e_{k} e_{l} d N_{d}$ $=0, k \neq l, \int \rho(s, t) e_{k}(s) d N_{d}(s)=\lambda_{k} e_{k}(t)$. The operator $B_{d}$ is of trace class with $\sum_{k \geq 1} \lambda_{k}=\int \rho(s, s) d N_{d}(s)<\infty$, and its Fredholm determinant $\delta_{d}$ is known to be $\delta_{d}(z)=\prod_{k \geq 1}\left(1-z \lambda_{k}\right), z \in \mathbb{C}$; see Dunford and Schwartz [6]. There is a version of the process $\left\{G(t), t \in \mathbb{R}^{d}\right\}$ which can be regarded as a Gaussian random element in the Hilbert space $L_{2}\left(\mathbb{R}^{d}, N_{d}\right)$. Its law is the same as that of $\sum_{k \geq 1} \sqrt{\lambda_{k}} e_{k} W_{k}$, where the $W_{k}$ are independent standard normal variables; see Araujo and Giné [2], page 157. It follows that $T=\int G^{2} d N_{d}$ has the same distribution as $\sum_{k \geq 1} \lambda_{k} W_{k}^{2}$. Its Laplace transform is $\delta_{d}(-2 t)^{-1 / 2}, t \geq 0$.

\section{The integral $\int G_{1}(t)^{2} d N_{d}(t)$}

Let $\rho=\rho_{1}$. To derive the complete system of eigenvalues and orthonormal eigenfunctions of the integral operator $B_{d}$, we introduce the integral operator $B_{0 d}$ : $L_{2}\left(\mathbb{R}^{d}, N_{d}\right) \rightarrow L_{2}\left(\mathbb{R}^{d}, N_{d}\right)$ defined by

$$
B_{0 d} f(y)=\int \exp \left(-\frac{1}{2}|x-y|^{2}\right) f(x) d N_{d}(x), \quad f \in L_{2}\left(\mathbb{R}^{d}, N_{d}\right), y \in \mathbb{R}^{d} .
$$

Let us treat the case $d=1$ first. For abbreviation, we put $N=N_{1}=N(0,1)$ and $b=\frac{1}{2}(\sqrt{5}-1)$. Note, that $b+1$ is the Golden Section number; see Vajda [11]. For verifying some of the subsequent assertions, it is useful to remember the identities $b^{2}=1-b, b=1 /(1+b), b^{2}=1 /(b+2)$. Denoting the Hermite polynomial of degree $n$ by $\mathcal{H} e_{n}(x)=(-1)^{n} \exp \left(x^{2} / 2\right) \frac{d^{n}}{d x^{n}} \exp \left(-x^{2} / 2\right), x \in \mathbb{R}$, we have

(5) $\frac{1}{\sqrt{2 \pi}} \int_{-\infty}^{+\infty} \exp \left(-\frac{1}{2}(x-y)^{2}\right) \mathcal{H} e_{n}(a x) d x=\left(1-a^{2}\right)^{n / 2} \mathcal{H} e_{n}\left(\frac{a y}{\left(1-a^{2}\right)^{1 / 2}}\right)$,

for any real $a,|a|<1$, and $y \in \mathbb{R}$ (see Erdélyi, Magnus, Oberhettinger and Tricomi [8], page 290, formula (17)). Starting from (5) and the orthogonality relations for the Hermite polynomials (see Erdélyi, Magnus, Oberhettinger and Tricomi [8], page 289 , formulas (9) and (11)) it can be verified that

$$
\gamma_{m}=b^{2 m+1}, \quad g_{m}(x)=\frac{5^{1 / 8}}{\sqrt{m !}} \mathcal{H} e_{m}\left(5^{1 / 4} x\right) \exp \left(-\frac{b}{2} x^{2}\right), \quad x \in \mathbb{R}, m=0,1, \ldots,
$$

is a complete system of eigenvalues and orthonormal eigenfunctions of $B_{01}$. In the case $d>1$ then obviously

$$
g_{d ; m_{1}, \ldots, m_{d}}\left(x_{1}, \ldots, x_{d}\right)=\prod_{j=1}^{d} g_{m_{j}}\left(x_{j}\right), \quad x_{j} \in \mathbb{R}, m_{j} \in\{0,1, \ldots\}, j=1, \ldots, d,
$$

is a complete system of orthonormal eigenfunctions of $B_{0 d}$. The eigenvalue associated with the eigenfunction $g_{d ; m_{1}, \ldots, m_{d}}$ is $\prod_{j=1}^{d} \gamma_{m_{j}}=b^{2\left(m_{1}+\cdots+m_{d}\right)+d}$. This means that the eigenvalues of $B_{0 d}$ are $b^{2 m+d}$ with multiplicity $\left(\begin{array}{c}m+d-1 \\ m\end{array}\right), m=0,1, \ldots$ Now 
we have that the operator $B_{d}$ is of the form $B_{d}=B_{0 d}-B_{1 d}$, where the operator $B_{1 d}: L_{2}\left(\mathbb{R}^{d}, N_{d}\right) \rightarrow L_{2}\left(\mathbb{R}^{d}, N_{d}\right)$ is defined by

$$
\begin{array}{r}
B_{1 d} f(y)=\left[\int \exp \left(-\frac{1}{2}|x|^{2}\right) f(x) d N_{d}(x)\right] \exp \left(-\frac{1}{2}|y|^{2}\right), \\
f \in L_{2}\left(\mathbb{R}^{d}, N_{d}\right), y \in \mathbb{R}^{d} .
\end{array}
$$

Again, let us consider the case $d=1$ first. For an odd function $f \in L_{2}(\mathbb{R}, N)$, the integral in (8) vanishes. Since the Hermite polynomials of degree $m=2 l+1, l=$ $0,1, \ldots$, are odd functions, it follows that the functions $g_{m}$ in (6) with $m=2 l+1$, $l=0,1, \ldots$, are also eigenfunctions of $B_{1}$. Therefore, $b^{4 l+3}, l=0,1, \ldots$, are also eigenvalues of $B_{1}$. To find the remaining eigenvalues of $B_{1}$ we argue as follows. A solution $g$ of

$$
B_{1} g=\gamma g
$$

for some positive $\gamma$, with $g$ being orthogonal to these $g_{2 l+1}$ for each $l=0,1, \ldots$, can be represented in the form $g=\sum_{l=0}^{\infty} a_{2 l} g_{2 l}$ with real $a_{2 l}$. The series converges in $L_{2}(\mathbb{R}, N)$. Putting

$$
b_{2 l}=\int \exp \left(-\frac{1}{2} x^{2}\right) g_{2 l}(x) d N(x)=5^{1 / 8} \frac{\sqrt{2 l} !}{2^{l} l !}(-1)^{l} b^{4 l+1}
$$

(see Abramowitz and Stegun [1], formula 22.13.17) and

$$
c=\int \exp \left(-\frac{1}{2} x^{2}\right) g(x) d N(x)
$$

we conclude, having such a solution of (9), that

$$
\sum_{l=0}^{\infty} a_{2 l} g_{2 l}\left(b^{4 l+1}-\gamma\right)=\sum_{l=0}^{\infty} c b_{2 l} g_{2 l} .
$$

It is immediately seen that $c \neq 0$ and that $\gamma \neq b^{4 l+1}, l=0,1, \ldots$ So, $a_{2 l}=$ $c b_{2 l} /\left(b^{4 l+1}-\gamma\right)$. Multiplying the left and the right hand sides of

$$
g(x)=\sum_{l=0}^{\infty} \frac{c b_{2 l}}{b^{4 l+1}-\gamma} g_{2 l}(x)
$$

by $\exp \left(-x^{2} / 2\right)$ and then integrating with respect to the standard normal distribution we get

$$
1=\sum_{l=0}^{\infty} \frac{b_{2 l}^{2}}{b^{4 l+1}-\gamma}
$$

Thus introducing the meromorphic function

$$
\omega_{1}(z)=1+5^{1 / 4} z \sum_{l=0}^{\infty} \frac{\frac{(2 l) !}{(l !)^{2} 2^{2 l}} b^{8 l+2}}{1-z b^{4 l+1}}, \quad z \in \mathbb{C},
$$

we find that $\xi=1 / \gamma$ is a zero of $\omega_{1}(z)$. On the other hand, having a zero $\xi$ of $\omega_{1}(z)$, it is easily seen that its inverse $1 / \xi$ is an eigenvalue of $B_{1}$ associated with the eigenfunction

$$
\sum_{l=0}^{\infty} \frac{b_{2 l}}{b^{4 l+1}-\gamma} g_{2 l}
$$


Although somewhat more intricate, the case $d>1$ can be treated in a similar manner. In fact, we get that for every $d$-tuple $\left(m_{1}, \ldots, m_{d}\right)$ with entries $m_{j} \in$ $\{0,1, \ldots\}$ at least one of which is odd, the function $g_{d ; m_{1}, \ldots, m_{d}}\left(x_{1}, \ldots, x_{d}\right)$ in $(7)$ is an eigenfunction of $B_{d}$ with associated eigenvalue $b^{2\left(m_{1}+\cdots+m_{d}\right)+d}$. On writing a further solution $g \in L_{2}\left(\mathbb{R}^{d}, N_{d}\right)$ of $B_{0 d}=\gamma g$ with associated eigenvalue $\gamma$ and being orthonormal to these $g_{d ; m_{1}, \ldots, m_{d}}\left(x_{1}, \ldots, x_{d}\right)$ in the form

$$
g=\sum_{m_{1}=0}^{\infty} \cdots \sum_{m_{d}=0}^{\infty} a_{2 m_{1}, \ldots, 2 m_{d}} g_{d ; 2 m_{1}, \ldots, 2 m_{d}}
$$

by analogy to (11) we get the identity

$$
\begin{gathered}
\sum_{m_{1}=0}^{\infty} \ldots \sum_{m_{d}=0}^{\infty} a_{2 m_{1}, \ldots, 2 m_{d}}\left(b^{4\left(m_{1}+\cdots+m_{d}\right)+d}-\gamma\right) g_{d ; 2 m_{1}, \ldots, 2 m_{d}} \\
\quad=\sum_{m_{1}=0}^{\infty} \cdots \sum_{m_{d}=0}^{\infty} c b_{2 m_{1}} \ldots b_{2 m_{d}} g_{d ; 2 m_{1}, \ldots, 2 m_{d}}
\end{gathered}
$$

where $c=\int \exp \left(-|x|^{2} / 2\right) g(x) d N_{d}(x)$, and the $b_{2 m_{j}}$ are defined in (10). Then we have to consider two cases.

Case 1: $\gamma=b^{4 m+d}$ for some nonnegative integer $m$. Then $c=0$, and $g$ is of the form

$$
g=\sum_{m_{1}+\cdots+m_{d}=m} a_{2 m_{1}, \ldots, 2 m_{d}} g_{d ; 2 m_{1}, \ldots, 2 m_{d}}
$$

with coefficients $a_{2 m_{1}, \ldots, 2 m_{d}}$ satisfying

$$
\sum_{m_{1}+\cdots+m_{d}=m} a_{2 m_{1}, \ldots, 2 m_{d}} b_{2 m_{1}} \ldots b_{2 m_{d}}=0
$$

The linear space of functions of this form is of dimension $\left(\begin{array}{c}m+d-1 \\ m\end{array}\right)-1$. So, $\gamma=b^{4 m+d}$ has multiplicity $\left(\begin{array}{c}m+d-1 \\ m\end{array}\right)-1$.

Case 2: $\gamma \neq b^{4 m+d}$ for every nonnegative integer $m$. Then $c \neq 0$, and $\gamma$ is obtained as a solution of the equation

$$
1=\sum_{m_{1}=0}^{\infty} \cdots \sum_{m_{d}=0}^{\infty} \frac{b_{2 m_{1}}^{2} \ldots b_{2 m_{d}}^{2}}{b^{4\left(m_{1}+\cdots+m_{d}\right)+d}-\gamma}=\sum_{m=0}^{\infty} 5^{d / 4}(-1)^{m}\left(\begin{array}{c}
-d / 2 \\
m
\end{array}\right) \frac{b^{8 m+2 d}}{b^{4 m+d}-\gamma}
$$

Alternatively, introducing the meromorphic function

$$
\omega_{d}(z)=1+5^{d / 4} z \sum_{m=0}^{\infty}(-1)^{m}\left(\begin{array}{c}
-d / 2 \\
m
\end{array}\right) \frac{b^{8 m+2 d}}{1-z b^{4 m+d}}
$$

we find that $1 / \gamma$ is a simple zero of $\omega_{d}(z)$. The associated function $g$ is

$$
\sum_{m_{1}=0}^{\infty} \cdots \sum_{m_{1}=0}^{\infty} \frac{b_{2 m_{1}} \ldots b_{2 m_{d}}}{b^{4\left(m_{1}+\cdots+m_{d}\right)+d}-\gamma} g_{d ; 2 m_{1}, \ldots, 2 m_{d}} .
$$

It is immediately seen that the $\gamma, g$ found are in fact solutions of $B_{d} g=\gamma g$.

Let us summarize the results obtained. To this end, note that for a given nonnegative integer $m$, the number of $d$-tuples $\left(m_{1}, \ldots, m_{d}\right)$ of nonnegative integers, at least one of which is odd and satisfies $m_{1}+\cdots+m_{d}=m$, is $\left(\begin{array}{c}m+d-1 \\ m\end{array}\right)-\left(\begin{array}{c}m / 2+d-1 \\ m / 2\end{array}\right)$ if $m$ is even and $\left(\begin{array}{c}m+d-1 \\ m\end{array}\right)$ if $m$ is odd. 
Theorem 1. Let $\xi_{m}, m=1,2, \ldots$, be the zeros of the meromorphic function $\omega_{d}(z)$. Then the distribution of $T_{1}=\int G_{1}(t)^{2} d N_{d}(t)$ is the same as that of

$$
\sum_{k=0}^{\infty} b^{4 k+d+2} \chi_{\left(\begin{array}{c}
2 k+d \\
2 k+1
\end{array}\right)}^{2}(k, 1)+\sum_{l=1}^{\infty} b^{4 l+d} \chi_{\left(\begin{array}{c}
(2 l+d-1 \\
2 l
\end{array}\right)-1}^{2}(l, 2)+\sum_{m=1}^{\infty} \frac{1}{\xi_{m}} \chi_{1}^{2}(m, 3),
$$

where the $\chi_{\left(\begin{array}{c}2 k+d \\ 2 k+1\end{array}\right)}^{2}(k, 1), \chi_{\left(\begin{array}{c}(2 l+d-1 \\ 2 l\end{array}\right)-1}^{2}(l, 2), \chi_{1}^{2}(m, 3)$ are independent $\chi^{2}$-variables, with the respective degrees of freedom indicated in the subscripts. The variables with subscript 0 occurring if $d=1$ are suppressed.

We deduce from Theorem 1 that the $r$ th cumulant of $T_{1}$ is

$$
\kappa_{r}=2^{r-1}(r-1) !\left\{\sum_{m=1}^{\infty}\left(\frac{1}{\xi_{m}}\right)^{r}+\left(\frac{b^{r}}{1-b^{2 r}}\right)^{d}-\frac{b^{r d}}{1-b^{4 r}}\right\}, \quad r \geq 1 .
$$

Arguing as in Darling [5] where a related problem is dealt with, it can be seen that the Fredholm determinant of $B_{d}$ is

$$
\left.\delta_{d}(z)=\omega_{d}(z) \prod_{m=0}^{\infty}\left(1-z b^{2 m+d}\right) \stackrel{(m+d-1}{m}\right), \quad z \in \mathbb{C} .
$$

This gives the representation $\left[1 / \delta_{d}(-2 t)\right]^{1 / 2}, t \geq 0$, for the Laplace transform of $T_{1}$. Expanding the rational expressions $1 /\left(1-z b^{4 m+d}\right)$ in (14) in geometric power series we get

$$
\omega_{d}(z)=\sum_{m=0}^{\infty} z^{m} /(F(2(m+1)))^{d / 2}, \quad|z|<b^{-d},
$$

with $F(m)$ the Fibonacci numbers determined by $F(m+1)=F(m)+F(m-1)$, $m=1,2, \ldots, F(0)=0, F(1)=1$. Using this and expanding $\log \left[1 / \delta_{d}(-2 t)\right]^{1 / 2}$ in powers of $t$, we see that the coefficient of $t^{r}$ is

$$
(-1)^{r} \frac{2^{r-1}}{r}\left\{\left(\frac{b^{r}}{1-b^{2 r}}\right)^{d}+\delta_{r}\right\}
$$

where

$$
\delta_{r}=r \sum_{k=1}^{r}(-1)^{k} \frac{1}{k} \sum_{\substack{m_{1}+\ldots+m_{k}=r \\ m_{i}=1,2, \ldots, i=1, \ldots, k}} \frac{1}{\left(F\left(2\left(m_{1}+1\right)\right) \ldots F\left(2\left(m_{k}+1\right)\right)\right)^{d / 2}} .
$$

Up to the multiplicative constant $(-1)^{r} r$ ! this coefficient is equal to the $r$ th cumulant of $T_{1}$. Since

$$
\frac{b^{r}}{1-b^{2 r}}= \begin{cases}1 /(\sqrt{5} F(r)) & \text { if } r \text { is even, } \\ 1 / L(r) & \text { if } r \text { is odd, }\end{cases}
$$

where the $L(r), r=0,1, \ldots$, are the Lucas numbers defined by $L(0)=2, L(1)=$ $1, L(r+1)=L(r)+L(r-1), r=1,2, \ldots$, the cumulants of $T_{1}$ can be written alternatively in the form

$$
\kappa_{r}= \begin{cases}2^{r-1}(r-1) !\left[(\sqrt{5} F(r))^{-d}+\delta_{r}\right] & \text { if } r \text { is even, } \\ 2^{r-1}(r-1) !\left[L(r)^{-d}+\delta_{r}\right] & \text { if } r \text { is odd. }\end{cases}
$$


Comparing (15) and (16) we are able to compute the infinite sums $\sum_{m=1}^{\infty} \xi_{m}^{-r}$, $r=1,2, \ldots$ In fact,

$$
\sum_{m=1}^{\infty}\left(\frac{1}{\xi_{m}}\right)^{r}=\frac{b^{r d}}{1-b^{4 r}}+\delta_{r} .
$$

The distribution of $T_{1}$ is most easily approximated by the distribution of the sum of finitely many of the weighted $\chi^{2}$-variables occurring in Theorem 1 . It is plain that such an approximation should be based on the sum consisting of the variables with the largest weights. So we have calculated by numerical methods the values of the 12 smallest zeros of $\omega_{1}(z)$ and $\omega_{2}(z)$. These values are shown in Table 1 .

TABLE 1 . The 12 smallest zeros $\xi_{i}, i=1, \ldots, 12$, of $\omega_{d}(z), d=1,2$

\begin{tabular}{r|c|c|}
\hline$\xi_{i}$ & $d=1$ & $d=2$ \\
\hline 1 & 7.849336555578469 & 9.980597866810863 \\
2 & 61.50441057386329 & 84.24682401892317 \\
3 & 448.1831773838836 & 638.7849329947641 \\
4 & 3179.147341949039 & 4645.090437279074 \\
5 & 22268.85055919773 & 33091.54159992883 \\
6 & 154929.5194062535 & 233046.4067592094 \\
7 & 1073517.332252734 & 1629672.896578112 \\
8 & 7419105.877262517 & 11343464.55464175 \\
9 & 51182811.59769955 & 78704778.23373327 \\
10 & 352654830.8574741 & 544825391.8014784 \\
11 & 2427578689.601553 & 3765058015.432077 \\
12 & 16699075379.06429 & 25984849966.06623 \\
\hline
\end{tabular}

The sum of all eigenvalues of $B_{d}$ is $\int\left(1-\exp \left(-|x|^{2}\right)\right) d N_{d}(x)=1-3^{-d / 2}$. For $d=1$, the sum of the eigenvalues $b^{4 m+3}, m=0,1, \ldots$, is $b / \sqrt{5}$. Adding to it the sum $\sum_{i=1}^{12} 1 / \xi_{i}$, where we use the calculated value stated in the $i$ th row of the first column of Table 1 for $\xi_{i}$, we get the value 0.422649730800 agreeing with the exact value $1-1 / \sqrt{3}$ up to 10 decimal places.

For $d=2$, the eigenvalues $b^{4 m+2}$ and $b^{4 m}$ both occur with multiplicity $2 m$, $m=1,2, \ldots$. The sum of these eigenvalues counted according to their multiplicities is $2 / 5+2 b^{2} / 5$. Adding to it the sum $\sum_{i=1}^{12} 1 / \xi_{i}$, where we use the calculated value stated in the $i$ th row of the second column of Table 1 for $\xi_{i}$, we get the value 0.666666666660 . The exact value is $2 / 3$.

\section{The integral $\int G_{2}(t)^{2} d N_{d}(t)$}

Let $\rho=\rho_{2}$. Let us treat the case $d=1$ first. From Mehler's expansion (see Erdélyi, Magnus, Oberhettinger and Tricomi [7], formula 10.13(22))

$$
\sum_{m=0}^{\infty} \frac{z^{m}}{m !} \mathcal{H} e_{m}(\sqrt{2} x) \mathcal{H} e_{m}(\sqrt{2} y)=\left(1-z^{2}\right)^{-1 / 2} \exp \left(\frac{2 x y z-\left(x^{2}+y^{2}\right) z^{2}}{1-z^{2}}\right)
$$


converging uniformly for $z$ in compact subsets of $(-1,+1)$, and for $x, y$ in compact subsets of $\mathbb{R}$, we get by putting $z=b^{2}, x=5^{1 / 4} s / \sqrt{2}$ and $y=5^{1 / 4} y / \sqrt{2}$ that

$$
\sum_{m=0}^{\infty} b^{2 m+1} g_{m}(s) g_{m}(t)=\exp \left(-(s-t)^{2} / 2\right) \text {. }
$$

Substituting $z=-b^{2}, x=5^{1 / 4} x / \sqrt{2}$ and $y=5^{1 / 4} t / \sqrt{2}$ in (17) we obtain

$$
\sum_{m=0}^{\infty}(-1)^{m} b^{2 m+1} g_{m}(s) g_{m}(t)=\exp \left(-(s+t)^{2} / 2\right) .
$$

Subtracting the expressions on the left (right) hand side of (19) from the expressions on the left (right) hand side of (18) we get the representation

$$
\sum_{m=0}^{\infty} b^{4 m+3} g_{m}(s) g_{m}(t)=\frac{1}{2}\left[\exp \left(-(s-t)^{2} / 2\right)-\exp \left(-(s+t)^{2} / 2\right)\right]
$$

From this we deduce that

$$
\sum_{m=0}^{\infty} b^{2 m+3 / 2} g_{m}(t) Z_{m}, \quad t \in \mathbb{R}
$$

is a version of $G_{2}$ with sample path in $L_{2}(\mathbb{R}, N)$. The square of its norm equals $\sum_{m=0}^{\infty} b^{4 m+3} Z_{m}^{2}$. Its distribution is the same as that of $T_{2}=\int G_{2}(t)^{2} d N(t)$.

Treating the case $d>1$ we put for abbreviation $e(u)=\exp \left(-u^{2} / 2\right), u \in \mathbb{R}$. Then the covariance function $\rho_{2}$ can be written as

$$
\rho_{2}(s, t)=\sum_{k=1}^{d} \frac{1}{2}\left[e\left(s_{k}-t_{k}\right)-e\left(s_{k}+t_{k}\right)\right] \prod_{j=1}^{k-1} e\left(s_{j}+t_{j}\right) \prod_{l=k+1}^{d} e\left(s_{l}-t_{l}\right),
$$

for $s=\left(s_{1}, \ldots, s_{d}\right)^{\prime} \in \mathbb{R}^{d}, t=\left(t_{1}, \ldots, t_{d}\right)^{\prime} \in \mathbb{R}^{d}$. In what follows we use the identities

$$
\begin{aligned}
& \int e(u-v) g_{m}(u) d N_{1}(u)=b^{2 m+1}, \quad m=0,1,2, \ldots, \\
& \int e(u+v) g_{m}(u) d N_{1}(u)= \begin{cases}b^{2 m+1} & \text { if } m \text { is even, } \\
-b^{2 m+1} & \text { if } m \text { is odd },\end{cases}
\end{aligned}
$$

and

$$
\int \frac{1}{2}[e(u-v)-e(u+v)] g_{m}(u) d N_{1}(u)= \begin{cases}0 & \text { if } m \text { is even } \\ b^{2 m+1} & \text { if } m \text { is odd. }\end{cases}
$$

Let $\mathbf{m}=\left(m_{1}, \ldots, m_{d}\right)$ be a $d$-tuple of nonnegative integers, and denote by $k(\mathbf{m})$ the number of its odd components $m_{i}$. It is easily verified that

$$
\int \rho_{2}(s, t) g_{d, m_{1}, \ldots, m_{d}}(t) d N_{d}(t)= \begin{cases}0 & \text { if } k(\mathbf{m}) \text { is even } \\ b^{2\left(m_{1}+\cdots+m_{d}\right)+d} g_{d ; m_{1}, \ldots, m_{d}}(s) & \text { if } k(\mathbf{m}) \text { is odd }\end{cases}
$$

If $\mathbb{M}^{d}$ is the set of all $d$-tuples of nonnegative integers with odd number $k(\mathbf{m})$, we see that $g_{d ; m_{1}, \ldots, m_{d}},\left(m_{1}, \ldots, m_{d}\right) \in \mathbb{M}^{d}$, is a collection of orthonormal eigenfunctions with associated positive eigenvalues

$$
b^{2\left(m_{1}+\cdots+m_{d}\right)+d}, \quad\left(m_{1}, \ldots, m_{d}\right) \in \mathbb{M}^{d} .
$$


The sum of these eigenvalues is

$$
\begin{aligned}
\sum_{\left(m_{1}, \ldots, m_{d}\right) \in \mathbb{M}^{d}} b^{2\left(m_{1}+\cdots+m_{d}\right)+d} & =\sum_{k=1}^{[(d+1) / 2]} \sum_{m=0}^{\infty} b^{4 m+d+4 k-2}\left(\begin{array}{c}
d \\
2 k-1
\end{array}\right)\left(\begin{array}{c}
m+d-1 \\
m
\end{array}\right) \\
& =\sum_{k=1}^{[(d+1) / 2]}\left(\begin{array}{c}
d \\
2 k-1
\end{array}\right) \frac{b^{2 d}}{\left(1-b^{4}\right)^{d}} b^{4 k-2-d} \\
& =5^{-d / 2} b^{-d} \frac{1}{2}\left[\left(1+b^{2}\right)^{d}-\left(1-b^{2}\right)^{d}\right] \\
& =\frac{1}{2}\left(1-5^{-d / 2}\right) .
\end{aligned}
$$

The sum of all positive eigenvalues of the integral operator $B_{d}$ is equal to $\int \rho_{2}(t, t) d N_{d}(t)$. An easy calculation yields $\int \rho_{2}(t, t) d N_{d}(t)=\frac{1}{2}\left(1-5^{-d / 2}\right)$. Therefore (21) gives the complete system of positive eigenvalues. Summarizing we can state the following result.

Theorem 2. The distribution of $T_{2}=\int G_{2}(t)^{2} d N_{d}(t)$ is the same as that of

$$
\sum_{k=1}^{[(d+1) / 2]} \sum_{m=0}^{\infty} b^{4 m+d+4 k-2} \chi_{(\underset{2 k-1}{d})\left(\begin{array}{c}
m+d-1 \\
m
\end{array}\right)}^{2},
$$

where the $\chi_{\left(\begin{array}{c}d \\ 2 k-1\end{array}\right)\left(\begin{array}{c}m+d-1 \\ m\end{array}\right)}^{2}$ are independent $\chi^{2}$-variables, with the degrees of freedom indicated in the subscripts.

By similar manipulations as those in (22) the $r$ th cumulant of $T_{2}$ is seen to be

$$
\begin{gathered}
2^{r-1}(r-1) ! \sum_{k=1}^{[(d+1) / 2]} \sum_{m=0}^{\infty} b^{(4 m+d+4 k-2) r}\left(\begin{array}{c}
d \\
2 k-1
\end{array}\right)\left(\begin{array}{c}
m+d-1 \\
m
\end{array}\right) \\
= \begin{cases}\frac{2^{r-2}(r-1) !}{F(2 r)^{d}}\left[(L(r) / \sqrt{5})^{d}-F(r)^{d}\right] & \text { if } r \text { is even, } \\
\frac{2^{r-2}(r-1) !}{F(2 r)^{d}}\left[F(r)^{d}-(L(r) / \sqrt{5})^{d}\right] & \text { if } r \text { is odd. }\end{cases}
\end{gathered}
$$

\section{Concluding REMARK}

The problem of testing the composite hypothesis of multivariate normality can be treated in a similar manner. But now in the case where the hypothesis is true the mean vector and covariance matrix of the true normal distribution are unknown and must be estimated from the data. It can be shown that a test statistic similar to (1) converges in distribution to the integral $\int G(t)^{2} d N_{d}(t)$ of a certain Gaussian process $\left\{G(t), t \in \mathbb{R}^{d}\right\}$ so that the methods of Section 2 apply. However, the covariance function of this process is more complicated. At present we are unable to give a complete solution analogous to those in Section 3 and Section 4.

\section{REFERENCES}

[1] M. Abramowitz and I. Stegun, Handbook of Mathematical Functions, Dover, New York, 1964. MR 29:4914

[2] A. Araujo and E. Giné, The Central Limit Theorem for Real and Banach Valued Random Variables, Wiley, New York, 1980. MR 83e:60003

[3] L. Baringhaus and N. Henze, A consistent test for multivariate normality based on the empirical characteristic function, Metrika 35 (1988), 339-348. MR 90f:62174 
[4] S. Csörgö, Multivariate empirical characteristic functions, Z. Wahrscheinlichkeitstheorie verw. Gebiete $5 \mathbf{5}$ (1981), 203-229. MR 82j:60026

[5] D. A. Darling, The Cramér-Smirnov test in the parametric case, Ann. Statist. 26 (1955), 1-20. MR 16:729g

[6] N. Dunford and J. T. Schwartz, Linear Operators, Part II, Wiley, New York, 1963. MR 32:6181

[7] A. Erdélyi, W. Magnus, F. Oberhettinger, and F. G. Tricomi, Higher transcendental functions. Vol. 2, McGraw-Hill, New York, 1953. MR 15:419i

[8] _ Tables of Integral Transforms. Vols. 1, 2, McGraw-Hill, New York, 1954. MR 15:868a; MR 16:468c

[9] A. Feuerverger and R. A. Mureika, The empirical characteristic function and its applications, Ann. Statist. 5 (1977), 88-97. MR 55:1605

[10] H.-D. Keller, Einige Untersuchungen zur empirischen charakteristischen Funtion und deren Anwendungen, Dissertation, Dortmund, 1979.

[11] S. Vajda, Fibonacci \& Lucas Numbers, and the Golden Section, Ellis Horwood, Chichester, 1989. MR 90h: 11014

[12] W. Whitt, Weak convergence of probability measures on the spaces $C[0, \infty)$, Ann. Math. Statist. 41 (1970), 939-944. MR 41:6259

Institut für Mathematische Stochastik, Universität Hannover, D-30167 Hannover, GERMANy

E-mail address: baringhaus@mbox.stochastik.uni-hannover.de 